\title{
ТЕЧЕНИЕ ОСЕСИММЕТРИЧНОЙ ИЗОТЕРМИЧЕСКОЙ СТРУИ ПРИ НЕВЫСОКИХ ЗНАЧЕНИЯХ ЧИСЛА РЕИНОЛЬДСА
}

\section{Введение}

Рост производства в последние десятилетия привел к значительному загрязнению биосферы. Поэтому защита окружающей среды от нежелательных последствий технического прогресса представляет собой часть общей проблемы охраны природы. Одним из источников загрязнения воздушного бассейна являются дымовые трубы крупных промышленных предприятий и ТЭС, выбросы из которых приводят к увеличению концентрации вредных примесей в приземном слое. Для прогнозирования опасного загрязнения воздуха разработаны математические модели с привлечением численных методов исследования и ЭВМ.

Определение загрязнения атмосферы из труб представляет собой аэродинамическую задачу струйного течения в сносящем потоке. Трудность ее решения заключается в том, что приходится учитывать все топографические и атмосферные факторы, влияющие на течение. Поэтому наряду с численными исследованиями проводят натурные измерения и масштабное моделирование. При масштабном моделировании необходимо сохранить одинаковыми критерии подобия на модели и объекте. Одним из критериев подобия является начальное число Рейнольдса $\mathrm{Re}_{0}$ на выходе из трубы. Известно, что развитие струи автомодельно при достаточно больших $\mathrm{Re}_{0}$, что позволяет уменьшить его значение при моделировании. Однако в области средних и низких $\mathrm{Re}_{0}$ такой автомодельности не наблюдается: за соплом появляется ламинарный участок, который влияет на распределение параметров в струе $\left[{ }^{1-4}\right]$. Для определения критического значения $\mathrm{Re}_{\mathrm{kp}}$, до которого существует автомодельность, и изучения влияния начального числа Рейнольдса на течение осесимметричной изотермической струи в области средних и низких значений было проведено настоящее экспериментальное исследование.

\section{Постановка эксперимента и методика измерения}

Изучалось распространение воздушной изотермической струи, вытекающей из круглого цилиндрического сопла $D_{0}=10$ мм при $\mathrm{Re}_{0}=$ $=600-14000$. Длина сопла составляла $100 D_{0}$, поэтому для всего диапазона значений $\mathrm{Re}_{0}$ на выходе из сопла получался характерный трубный профиль скорости. Струя в поперечном потоке исследовалась в аэродинамической трубе $\left[{ }^{5}\right]$. Безразмерный комплекс $I$, характеризующий влияние начального импульса на форму оси струи, принимал значения $1-36$. Начальное число Рейнольдса определялось по средней скорости $\bar{u}$ на выходе из сопла: 


$$
\mathrm{Re}_{0}=\bar{u} D_{0} / v,
$$

где

$$
\bar{u}=\int_{F} \varrho u d F / \varrho_{\mathrm{c}} F .
$$

Поле скорости затопленной струи измерялось трубкой Прандтля-Пито, а струи в поперечном потоке - 3-канальным зондом. Полученные величины фиксировались электрическим микроманометром «Furness controll Limited MDC» с нижним пределом измерения до 0,004 Wg, что позволяло экспериментировать при небольших $\mathrm{Re}_{0}$ с большой степенью точности. Погрешность не превышала $2 \%$. Для качественного анализа струю фотографировали.

\section{Результаты опытов и их обсуж'дение}

3 атопленная струя. При низких значениях $\mathrm{Re}_{0}$ (рис. 1) за соплом образуется участок, где смешение струи с окружающей средой

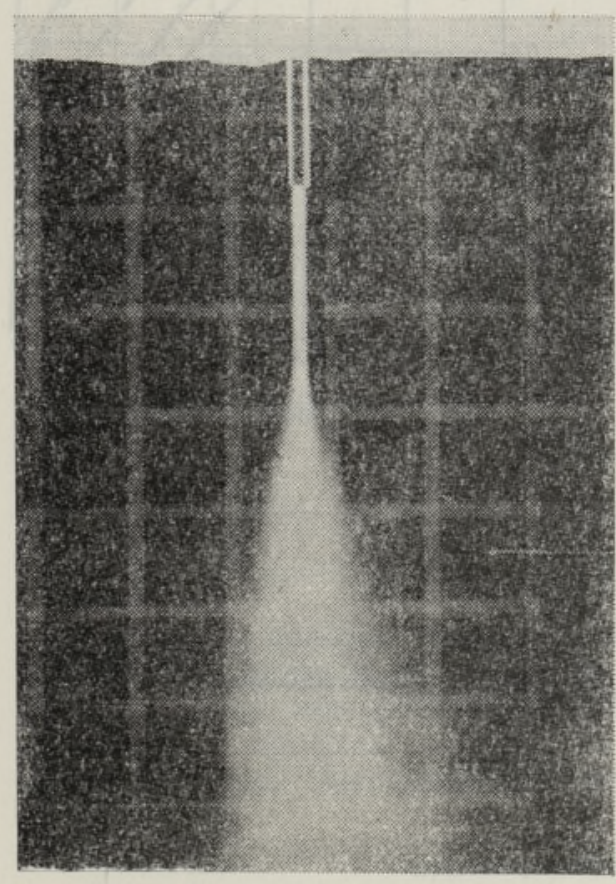

Рис. 1. Затопленная струя при $\mathrm{Re}_{0}=900$. происходит в основном за счет молекулярной диффузии. На некотором расстоянии от сопла струя становится турбулентной. Процесс перехода к турбулентности сложен, состоит из нескольких этапов [ $\left.{ }^{1}\right]$. Условно течение струи с неравномерным (трубным) профилем скорости на выходе из сопла можно разделить на три участка (рис. 2).

Первый участок - ламинарный. Профиль скорости почти не деформируется, струя практически не расширяется, осевая скорость остается неизменной по всему участку, длина которого зависит от $\mathrm{Re}_{0}$ и может достигать значений больше $10 D_{0}$ (рис. 3 ). С увеличением $\mathrm{Re}_{0}$ участок уменьшается и $\mathrm{B}$ случае приближения к $\mathrm{Re}_{\mathrm{kp}}=3000$ исчезает.

Второй участок - переходный. Профиль скорости начинает деформироваться, струя расширяться. Длина переходного участка с ростом $\mathrm{Re}_{0}$ до критического увеличивается, а при $\mathrm{Re}_{0}$, больше критического, изменяется мало. Роль переходного участка очень существенна в струйном течении, так как здесь значительно затухает осевая скорость, причем интенсивность затухания зависит от $\mathrm{Re}_{0}$.

Третий участок - основной. Профиль скорости становится автомодельным.

Профиль скорости на выходе из сопла в зависимости от $\mathrm{Re}_{0}$ показан на рис. 4. При $\mathrm{Re}_{0}>3000$ профиль скорости остается неизменным, что характерно для турбулентного режима. С уменьшением $\mathrm{Re}_{0}$ он начинает резко изменяться и при $\mathrm{Re}_{0}=600$ приближается к ламинарному: 


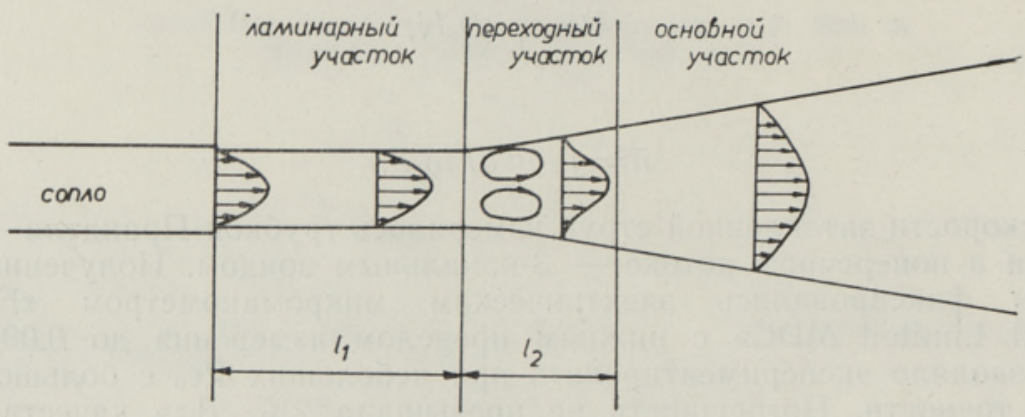

Pıс. 2. Схема затопленной струи.

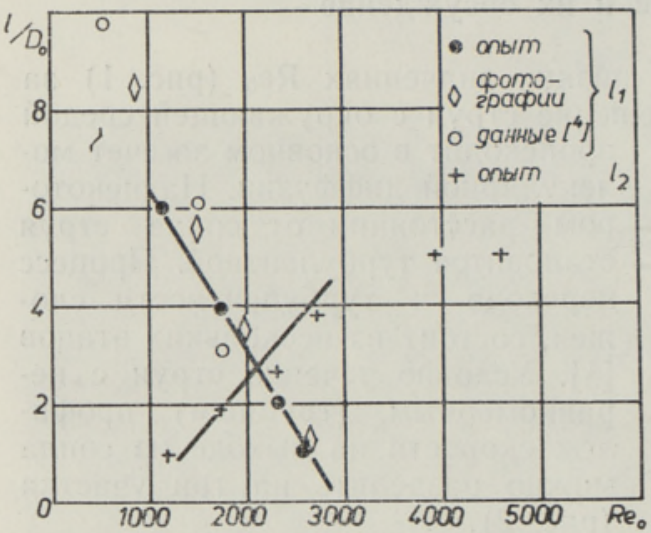

Рис. 3. Длина ламинарного и переходного участков.

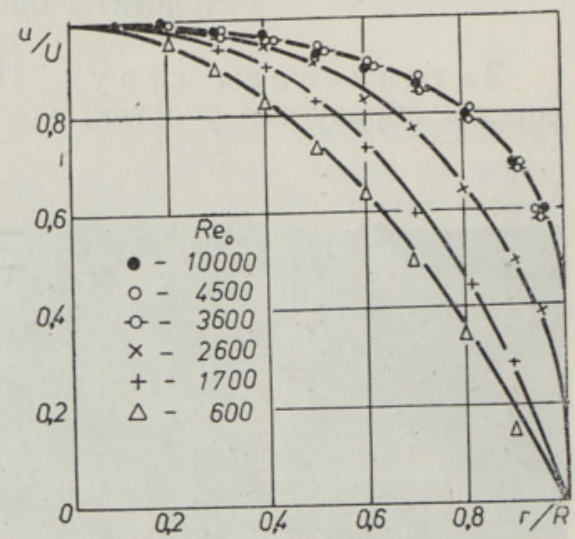

Рис. 4. Профиль скорости.

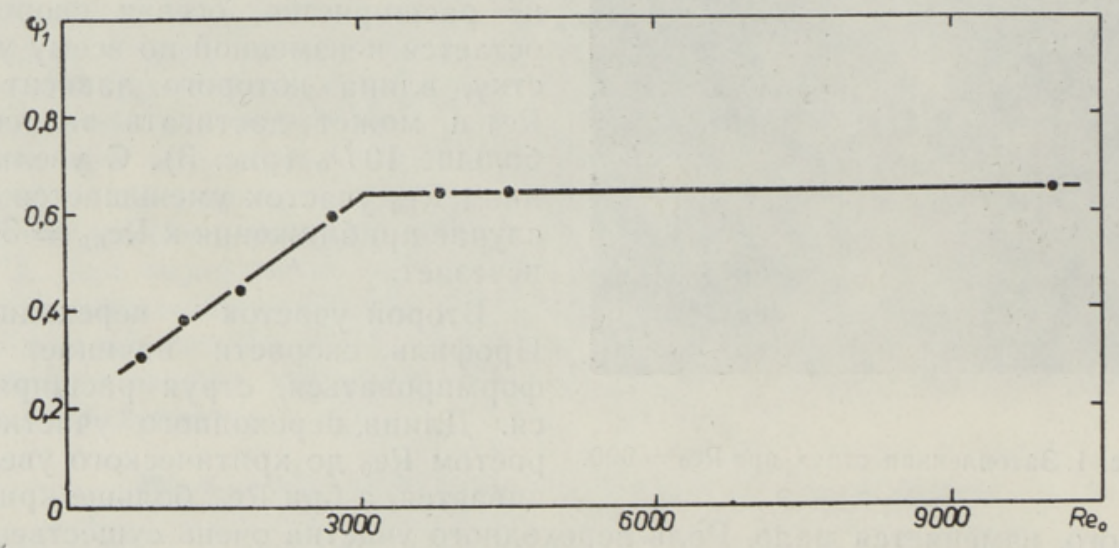

Рис. 5. Деформация профиля.

$$
u / U=1-(r / R)^{2} .
$$

Если безразмерный профиль скорости представить зависимостью

$$
u / U=(1-r / R)^{1 / m},
$$

то при $\mathrm{Re}_{0}>3000$ значение $m$ меняется слабо, оставаясь близким к 


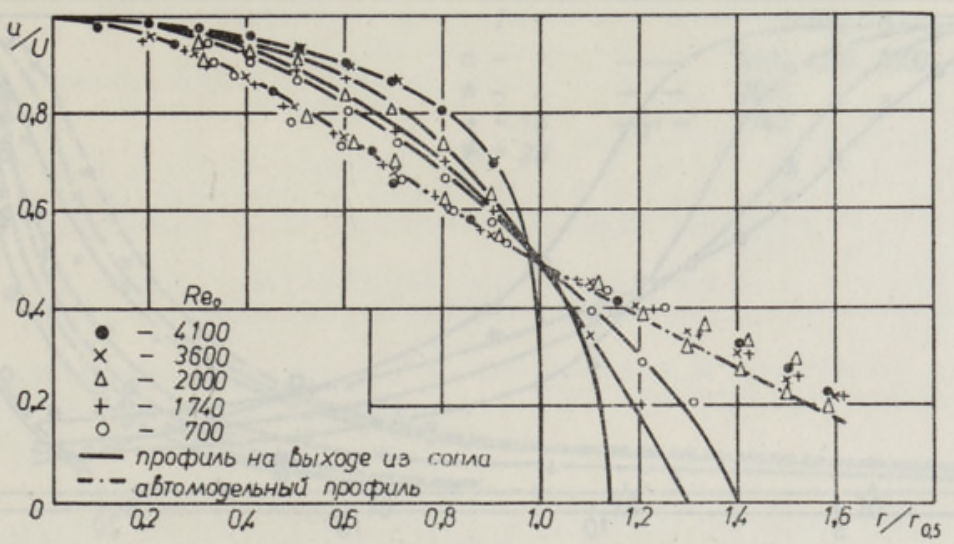

Рис. 6. Универсальный профиль.

семи. При $600 \leqslant \mathrm{Re}_{0} \leqslant 3000$ изменение $m$ можно представить эмпирической зависимостью

$$
m=\mathrm{Re}_{0} / 500+1 \text {. }
$$

Формула (5) в случае приближения профиля к ламинарному дает по сравнению с равенством (3) погрешность, практически не влияющую на интегральный расчет струи.

Другим параметром, характеризующим деформацию профиля скорости, является коэффициент поля $\psi_{1}$ (рис. 5), который определяется по динамическому напору

где

$$
\psi_{1}=\bar{u}^{2} / U^{2},
$$

$$
\bar{u}^{2}=2 \int_{0}^{R} u^{2} r d r / R^{2} .
$$

На рис. 6 показан построенный по «собственной» координате универсальный профиль на основном участке и профили скорости на выходе из сопла при различных значениях $\mathrm{Re}_{0}$. Следует отметить, что ламинарный профиль скорости по своей форме близок к универсальному профилю. Последний хорошо аппроксимируется известной зависимостью [6]

где

$$
u / U=1-6 \eta^{2}+8 \eta^{3}-3 \eta^{4}
$$

$$
\eta=r / r_{0,5}
$$

Затухание осевой скорости при различных числах $\operatorname{Re}_{0}$ показано на рис. 7. При $\mathrm{Re}_{0}>3000$, т. е. при турбулентном профиле на выходе из сопла, затухание скорости автомодельно по $\mathrm{Re}_{0}$. При $600 \leqslant \mathrm{Re}_{0} \leqslant 3000$ осевая скорость на ламинарном участке остается постоянной, а на переходном начинает резко уменьшаться, причем интенсивность затухания сильно зависит от $\mathrm{Re}_{0}$. При аппроксимации затухания скорости по оси степенной зависимостью

$$
U / U_{0} \sim x^{-\mathrm{K}_{u}}
$$

коэффициент затухания $\kappa_{u}$ на основном участке равен единице. Из рис. 7 видно, что наиболее важную роль играет переходный участок. 


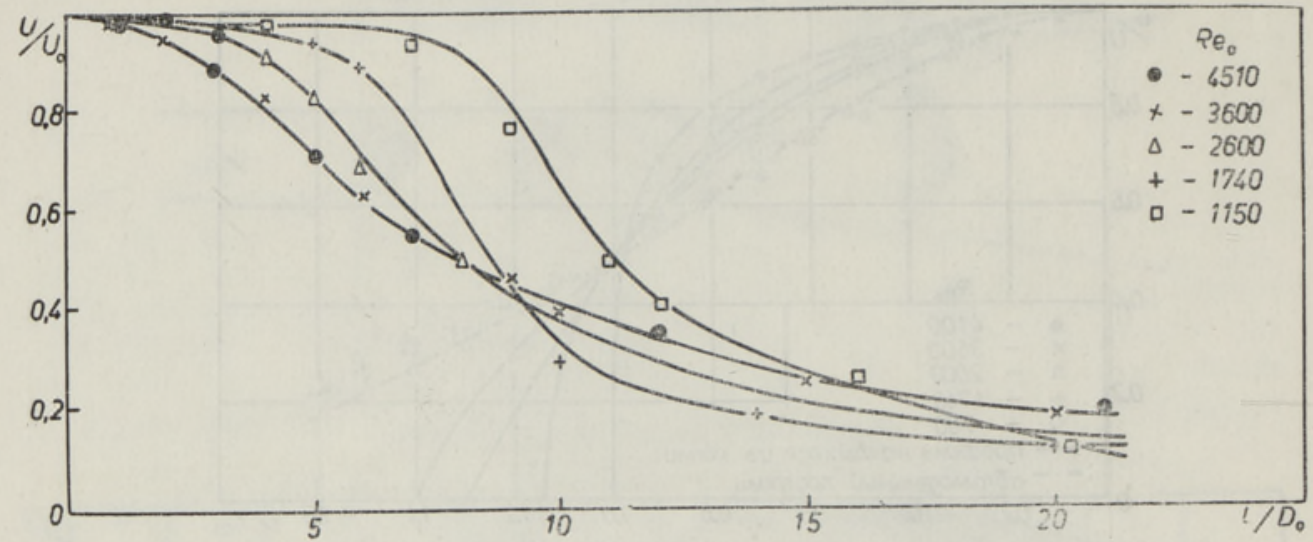

Рис. 7. Затухание осевой скорости.

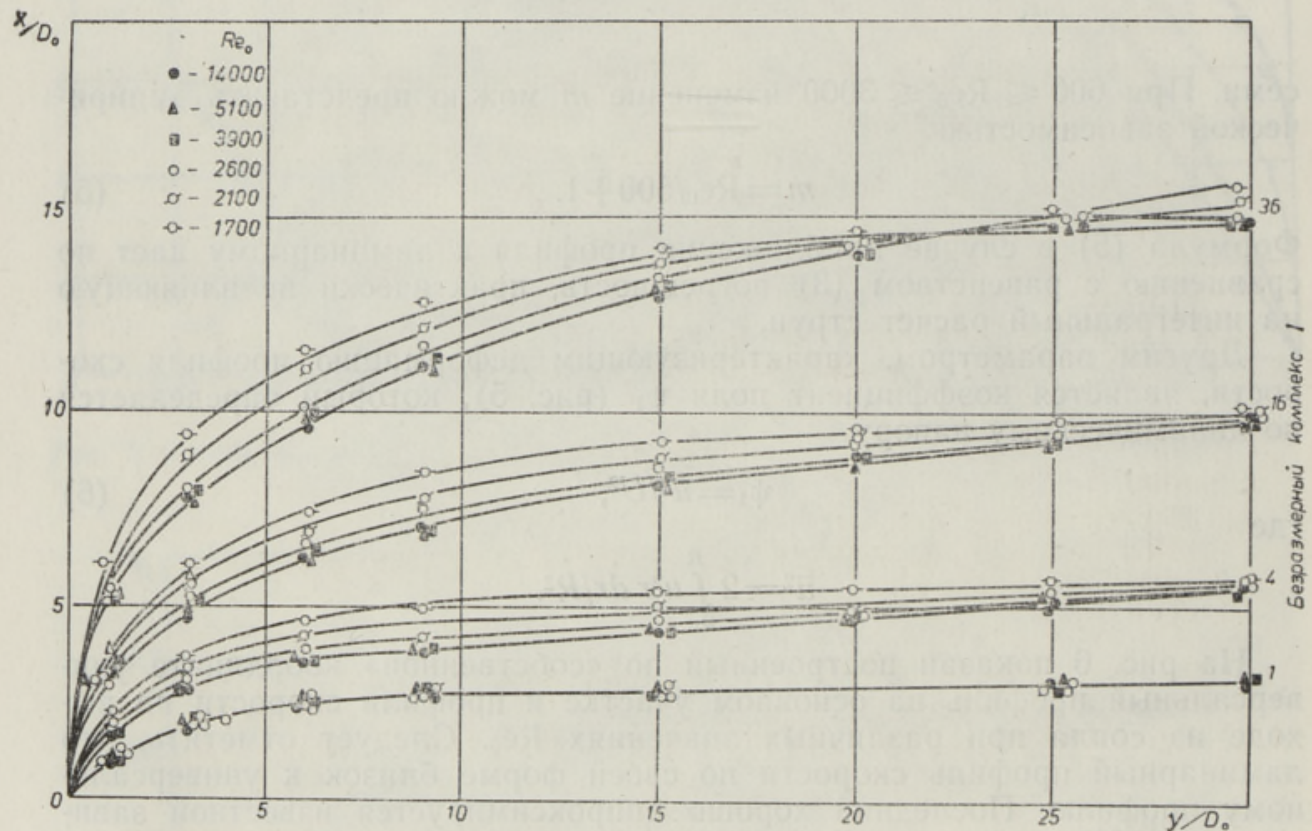

Рис. 8. Траектория струи.

С тр уя в поперечном потоке. Особенности развития затопленной струи, связанные с появлением ламинарного участка, характерны и для развития струи в поперечном потоке. Учитывая сложную структуру струи в поперечном потоке и ее недостаточную изученность, в эксперименте определялось влияние $\mathrm{Re}_{0}$ на траекторию струи и на затухание осевой избыточной скорости. Траектория струи определялась как геометрическое место точек с максимальной скоростью в поперечных сечениях. При $\mathrm{Re}_{0}>3000$ форма оси струи не зависит от $\mathrm{Re}_{0}$ (рис. 8) и является функцией только от двух параметров: $Y / D_{0}=f\left(X / D_{0} ; I\right)$. При $\mathrm{Re}_{0}$ ниже критического положение оси струи заметно изменяется и определяется как функция $Y / D_{0}=f\left(X / D_{0} ; I ; \mathrm{Re}_{0}\right)$. B поперечном потоке ламинарный участок в струе заметно меньше, чем у затопленной. При $I \sim 1$ форма оси струи при всех значениях $\mathrm{Re}_{0}$ оди- 


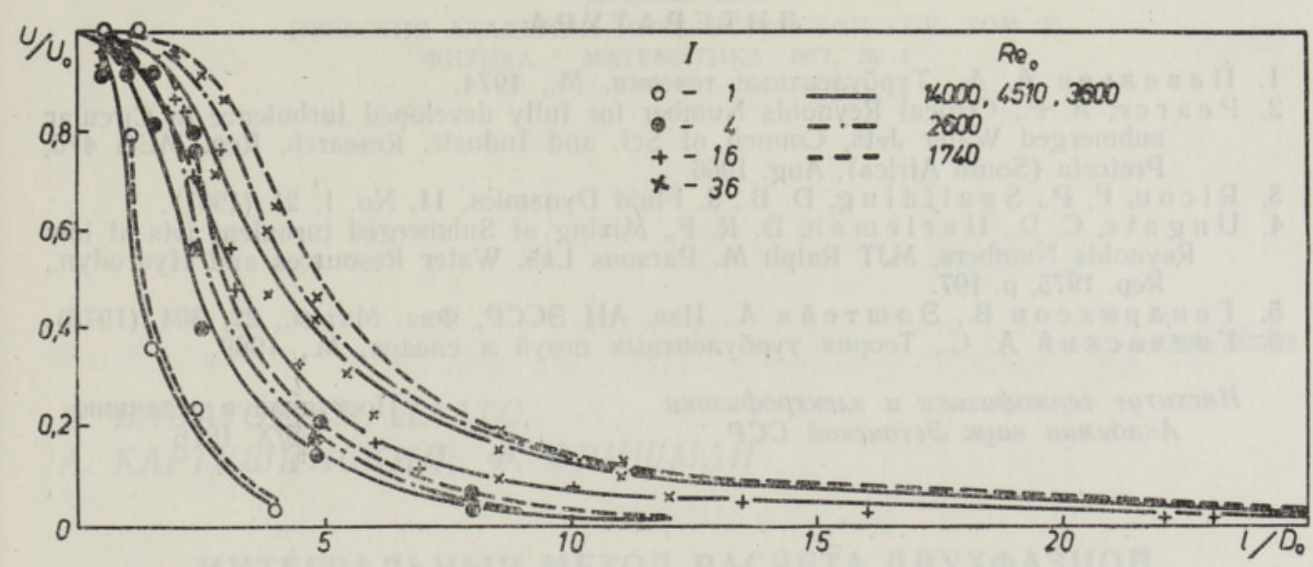

Рис. 9. Затухание осевой избыточной скорости.

накова, причем ламинарного участка практически нет. С ростом $I$ увеличивается длина ламинарного участка и изменяется форма оси струи на выходе из трубы. Ось струи с ламинарным участком вблизи сопла проходит выше, чем у полностью турбулентной струи, а на удалении от него (50-100 $\left.D_{0}\right)$, как показывают фотографии струи, - несколько ниже. Это объясняется более интенсивным затуханием осевой избыточной скорости у струи с ламинарным участком (рис. 9), которая приближается к направлению набегающего потока раньше полностью турбулентной, а осевая скорость быстрее стремится к его скорости.

\section{Выводы}

1. В области жевысоких значений числа Рейнольдса существует $\mathrm{Re}_{\text {кр }}$, ниже которого в струе появляется ламинарный участок и отсутствует автомодельность.

2. Наличие ламинарного участка значительно влияет на интенсивность перемешивания струи с окружающей средой.

3. При моделировании с невысокими значениями числа Рейнольдса необходимо учитывать влияние $\mathrm{Re}_{0}$ на течение струи.

\section{Обозначения}

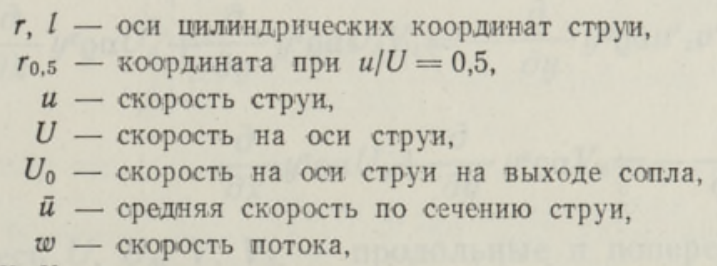

$X, Y$ - оси декартовой системы координат для струи в поперечном потоке,

$\mathrm{Re}_{0}$ - значение числа Рейнольдса на выходе из трубы,

$D_{0}=2 R$ - начальный диаметр сопла,

$I$ - безразмерный комплекс $=\bar{u}^{2} / w^{2}$,

$v$ - кинематический коэффициент молекулярной вязкости,

Q - плотность струи,

Qc - средняя плотность по сечению струи,

$F$ - площадь поперечного сечения струи, 
Л ИТЕРА Т У Р А

1. Павельев А. А., Турбулентные течения, М., 1974.

2. P e a r ce, A. F., Critical Reynolds Number for fully developed turbulence in Circular submerged Water Jets, Council of Sci. and Industr. Research, Rep. MEG 475; Pretoria (South Africa), Aug. 1966.

3. Ricou, F. P., S p alld ing, D. B., J. Fluid Dynamics, 11, No. 1, 21 (1961).

4. Ungate, C. D., H a r l em a n, D. R. F., Mixing of Submerged turbulent jets at low Reynolds Numbers, MJT Ralph M. Parsons Lab. Water Resources and Hydrodyn., Rep. 1975, p. 197.

5. Генд риксон В., Эпштейн А., Изв. АН ЭССР, Физ. Матем., 22, 304 (1973).

6. Гинев ский А. С., Теория турбулентных струй и следов, М., 1969.

Ннститут термофизики и электрофизики Академии наук Эстонской ССР
Поступила в редакцию $5 / \mathrm{X} 1976$

\section{VINOGRADOV}

\section{TELGSOMMEETRILISE ISOTERMILISE JOA VOOLAMINE VÄIKESTE REYNOLDSI ARVUDE PUHUL}

On uuritud algse Reynoldsi arvu (600-14000) mõju vabale joale ja ristvooluses olevale joale. Selgub, ct seoses $\operatorname{Re}_{o} \leqslant \operatorname{Re}_{\mathrm{kr}} \leqslant 3000$ vähenemisega tekib $\mathrm{Re}_{\mathrm{o}}$ järgi isemodelteeruvuse häire. On tehtud kindlaks ristvooluses oleva joa laminaarpiirkonna pikkuse, teigkiiruse sumbumiste, kiiruse profiilide ja joa telje vormide sōltuvus $\mathrm{Re}_{0}$-st, kui $\mathrm{Re}_{\mathrm{o}} \leqslant \mathrm{Re}_{\mathrm{kr}}$. Kui $\mathrm{Re}_{\mathrm{o}}>\mathrm{Re}_{\mathrm{kr}}$, toimub isemodelleerumine $\mathrm{Re}_{\mathrm{o}}$ järgi.

\section{VINOGRADOV}

\section{THE FLOW OF AN AXISYMMETRIC ISOTHERMAL JET AT LOW VALUES OF REYNOLDS NUMBER}

An experimental study of the dependence of the flow in a submerged jet and a jet in the cross stream on Reynolds number $\operatorname{Re}_{o}(600-14000)$ has been carried out. The independence of the flow pattern is shown to be broken at $\mathrm{Re}_{\mathrm{o}} \leqslant \mathrm{Re}_{\text {crit }} \leqslant 3000$. The dependence of the laminar section length, axial velocity decay, velocity profile and the trajectory of a jet in the cross stream on $\mathrm{Re}_{o}$ has been proved. At $\operatorname{Re}_{0}>\operatorname{Re}_{\mathrm{crit}}$ the flow pattern is independent from $\mathrm{Re}_{0}$. 